Learning Objectives:

Objectives / Hypothesis: To observe the usefulness of anterior based periosteal (Palva) flap for mastoid cavity obliteration in canal wall down tympanomastoidectomy and review its efficacy in producing a dry, low-maintenance, small mastoid cavity.

Study design: Retrospective study of a consecutive series of procedures from 2012 to 2014.

Methods: Sixty one consecutive procedures for active chronic otitis media with a minimum follow-up of 6 months (mean, $21 \mathrm{mo}$; range, 6-40 mo).

Results: 45 ears of cholesteatoma and 11 ears of adhesive otitis media were enrolled this study, and others were chronic otitis media(4 ears), adenoma of middle ear( 1 ear). 52 ears $(85.2 \%)$ maintained a small, dry, healthy mastoid cavity. 3 ears (4.9\%) had intermittent otorrhea easily controlled by topical treatment, 2 ears $(3.2 \%)$ had persistent otorrhea. 3 ears $(4.9 \%)$ had showed reperforation of tympanic membrane. There were 1 ears of residual or recurrent cholesteatomas. Outcomes remained stable over progressively longer follow-up, up to 40 months.

Conclusion: Obliteration of a canal wall down mastoid cavity by a postauricular periosteal flap is a reliable and effective technique that results in a dry, trouble-free mastoid cavity in $85.2 \%$ of patients with active chronic otitis media.

doi:10.1017/S0022215116006009

\section{ID: IP104}

Reliability and comparison of gain values with occurrence of saccades in the video head impulse test (vHIT)

\section{Presenting Author: Leise Hviid Korsager}

Leise Hviid Korsager ${ }^{1}$, Jens Hoejberg Wanscher ${ }^{2}$, Jesper Hvass Schmidt ${ }^{3}$, Christian Faber ${ }^{2}$

${ }^{1}$ Odense University Hospital, ${ }^{2}$ Department of ENT-Head \& Neck Surgery, Odense University Hospital, ${ }^{3}$ Department of Audiology, Odense University Hospital

\section{Learning Objectives:}

Introduction: The vHIT investigates the vestibular function in two ways: a VOR (vestibulo-ocular reflex) gain value and a graphical representation of VOR. Interpreting patient's vestibular function based on vHIT depends on both parameters, but more information about the reliability of the two parameters is needed.

The objective was to investigate the reliability of vHIT by comparing gain values between examiners on the same subjects and to see how differences affected the occurrence of saccades.

Method: Thirty subjects who had undergone cochlear implant (CI) surgery, were tested with video head impulse test (EyeSeeCam from Interacoustics). Four different examiners, all experienced with vHIT, tested the subjects.

Two judges interpreted the graphical representation of VOR according to occurrence of saccades or not.
Results: Differences in gain values amongst examiners varied from $0.2-0.58$ with an average of $0.14(95 \%$ CI $0.12-0.16)$ on the right ear and $0.17(95 \%$ CI $0.15-0.19)$ on the left ear. Occurrences of saccades on the same patient were reproduced in $93 \%$ of the cases by all examiners. Interclass correlation coefficient (ICC) of the gain values between two examiners was 0.62. Kappa's coefficient was calculated upon the interpretation of the graphical outcome to 0.83 .

Conclusion: The gain value seems to be less reliable than the graphical occurrence of saccades in the judgement of VOR. Interpretation of vHIT results should therefore not depend on the gain value alone but should depend on both gain value and the occurrence of saccades.

doi:10.1017/S0022215116006010

\section{ID: IP105}

Vestibular findings after cochlear implant surgery measured by video head impulse test (vHIT): A double blinded, randomised clinical trial

\section{Presenting Author: Leise Hviid Korsager}

Leise Hviid Korsager ${ }^{1}$, Jens Hoejberg Wanscher ${ }^{2}$, Jesper Hvass Schmidt ${ }^{3}$, Christian Faber ${ }^{2}$

${ }^{1}$ Odense University Hospital, ${ }^{2}$ Department of Rhinolaryngology, Odense University Hospital, ${ }^{3}$ Department of Audiology, Odense University Hospital

\section{Learning Objectives:}

Introduction: Dizziness is a common side effect to cochlear implant (CI) surgery. Regarding the CI surgical technique, there is no clear evidence if one approach (round window approach) leads to less dizziness than another approach (cochleostomy)

The main objective to this study is to investigate any difference between the two surgical approaches measured by video head impulse test (vHIT). Secondly we compare the objective findings with the subjective dizziness perceived by the patient.

Method: Fifty patients who will undergo CI surgery at OUH will be examined with vHIT prior to their surgery, the day after their surgery and one month after. They will fill out a Dizziness Handicap Inventory (DHI) scheme and VAS score according to their dizziness.

Subjects are randomized to either the round window approach or the cochleostomy approach. Subjects are stratified according to age $(+/-60)$, hearing rest and gain prior to surgery $(+/-0,68)$. The randomization is blinded for investigator and subject.

Inclusion period ends at $1^{\text {st }}$ of April 2016.

Results: Results will be revealed at the conference.

Conclusion: The results of this study could have influence on the future choice of approach of electrode insertion in cochlear implant surgery. 